Comment. Math. Helv. 72 (1997) 1-15 0010-2571/97/010001-15\$1.50+0.20/0
(C) 1997 Birkhäuser Verlag, Basel

Commentarii Mathematici Helvetici

\title{
Cohomologie des groupes et corps d'invariants multiplicatifs tordus
}

\author{
Jean Barge
}

Abstract. We give a cohomological calculation of the unramified Brauer group of a field of invariants under a twisted multiplicative action of a finite group.

Mathematics Subject Classification (1991). 12Gxx.

Keywords. Cohomology of groups, Brauer group, multiplicative fields of invariants.

Dans [S3], Saltman détermine le groupe de Brauer non ramifié des corps d'invariants d'actions multiplicatives tordues.

Précisément, soient $G$ un groupe fini et $M$ un $G$ réseau - i.e. un $\mathbf{Z}$-module libre et de type fini sur lequel $G$ opère fidèlement - et enfin soit $f: G \rightarrow \widehat{M}$ un homomorphisme croisé du groupe $G$ dans le $G$-module $\widehat{M}=\operatorname{Hom}_{\mathbf{Z}}\left(M ; \mathbf{C}^{*}\right)$. On fait opérer le groupe $G$ sur l'anneau de groupe $\mathbf{C}[M]$ par la formule

$$
g \circ X^{m}=f(g)(g . m) X^{g \cdot m} .
$$

Nous noterons $\mathbf{C}[M]_{f}\left(\operatorname{resp} \mathbf{C}(M)_{f}\right)$ cet anneau de groupe muni de l'action précédente (resp son corps des fractions muni de l'action induite).

Il est important de savoir calculer le groupe de Brauer non ramifié du corps des invariants $\mathbf{C}(M)_{f}^{G}$, car la non-nullité de ce groupe est la première obstruction à la rationalité stable [S1][S2].

Notons $\mathbf{C}[M]_{f}^{*}$ le $G$-module des unités de l'anneau $\mathbf{C}[M]_{f}$ de sorte que l'on a la suite exacte (non généralement scindée) de $G$-modules

$$
0 \rightarrow \mathbf{C}^{*} \stackrel{i}{\rightarrow} \mathbf{C}[M]_{f}^{*} \rightarrow M \rightarrow 0 .
$$

Notons $\beta_{G}$ l'ensemble des homomorphismes du groupe $\mathbf{Z} \times \mathbf{Z}$ dans $G$ et $\widetilde{\beta}_{G}$ le sous-ensemble de $\beta_{G}$ constitué des homomorphismes $\rho$ pour lesquels l'application

$$
i_{*}: H^{2}\left(\operatorname{Im} \rho ; \mathbf{C}^{*}\right) \rightarrow H^{2}\left(\operatorname{Im} \rho ; \mathbf{C}[M]_{f}^{*}\right)
$$

est injective. Nous sommes maintenant en mesure d'énoncer le théorème de Saltman [S3]. 
Théorème 1. Le groupe de Brauer non ramifié $B r^{n r}\left(\mathbf{C}(M)_{f}^{G}\right)$ du corps d'invariants $\mathbf{C}(M)_{f}^{G}$ est isomorphe au noyau des homomorphismes de restrictions:

$$
H^{2}\left(G ; \mathbf{C}[M]_{f}^{*}\right) \longrightarrow \prod_{\rho \in \widetilde{\beta}_{G}} H^{2}\left(\operatorname{Im} \rho ; \mathbf{C}[M]_{f}^{*}\right)
$$

Le présent article, qui fait suite à [Ba] contient d'abord une nouvelle démonstration du théorème précédent dans le cas particulier où le $G$-réseau $M$ est d'indice fini dans un $G$-réseau de permutation. Comme dans [Ba] on exploite le fait que les actions multiplicatives - même tordues - se linéarisent et l'ingrédient fondamental est bien sûr le théorème démontré indépendamment par Bogomolov et Saltman qui détermine le groupe de Brauer non ramifié du corps d'invariants d'une action linéaire.

Théorème 2. [Bo] Soit $\Gamma$ un groupe fini opérant linéairement et fidèlement sur un $\mathbf{C}$-espace vectoriel $V$. Le groupe de Brauer non ramifié $\operatorname{Br}^{n r}\left(\mathbf{C}(V)^{\Gamma}\right)$ du corps d'invariants $\mathbf{C}(V)^{\Gamma}$ est égal au noyau des homomorphismes de restriction:

$$
H^{2}\left(\Gamma ; \mathbf{C}^{*}\right) \longrightarrow \prod_{\rho \in \beta_{\Gamma}} H^{2}\left(\operatorname{Im} \rho ; \mathbf{C}^{*}\right) .
$$

(voir aussi [C.T])

Le théorème ci-dessus admis, la méthode est la suivante.

Supposons le $G$-réseau $M$ d'indice fini dans un $G$-réseau de permutations $P$ de sorte que l'on a les inclusions de corps

$$
\mathbf{C}(M)_{f}^{G} \subset \mathbf{C}(M)_{f}=\mathbf{C}(M) \subset \mathbf{C}(P) .
$$

On montre les faits suivants.

a) L'extension $\mathbf{C}(P) / \mathbf{C}(M)_{f}^{G}$ est galoisienne.

b) Son groupe de Galois $\Gamma$, agit linéairement sur $\mathbf{C}(P)$.

c) On a la suite exacte :

$$
0 \rightarrow \widehat{F} \rightarrow \Gamma \rightarrow G \rightarrow 1
$$

où $F$ est le conoyau de $M$ dans $P$ et cette extension est déterminée par la classe $[f] \in H^{1}(G ; \widehat{M})$ de l'homomorphisme croisé $f$.

On a donc $\mathbf{C}(P)^{\Gamma}=\mathbf{C}(M)_{f}^{G}$ et a fortiori $B r^{n r}\left(\mathbf{C}(P)^{\Gamma}\right)=B r^{n r}\left(\mathbf{C}(M)_{f}^{G}\right)$.

On applique alors le théorème 2 à $\Gamma$.

La méthode précédente est d'ailleurs réversible, si bien qu'on peut considérer ce papier, soit comme une nouvelle détermination des groupes de Brauer non ramifiés de corps d'invariants multiplicatifs tordus, soit comme un calcul du groupe 
de Brauer non ramifié du corps d'invariants d'une action linéaire d'un groupe $\Gamma$, extension quelconque d'un groupe fini par un groupe abélien fini.

Le deuxième paragraphe de cet article est purement cohomologique. Il est consacré à la constatation que les résultats des théorèmes 1 et 2 sont bien en accord avec l'égalité (1). C'est donc une nouvelle démonstration du théorème 1 , à partir du théorème 2. Néanmoins, il semble bien que pour les calculs "effectifs" l'expression donnée par le théorème 2 soit plus maniable. En particulier, il est facile sous cette forme d'obtenir des exemples d'actions multiplicatives tordues du groupe $\mathbf{Z} / p \times \mathbf{Z} / p$ pour $p$ premier quelconque dont le groupe de Brauer non ramifié du corps d'invariants soit non nul, et donc a fortiori non stablement transcendant pur. Voir aussi [S3].

La construction de cet exemple occupe le paragraphe 3 .

Ce fait contraste avec le théorème de [Ba] qui affirme qu'un tel exemple n'existe pas pour des actions multiplicatives non tordues.

En fait nous avions montré :

Théorème [Ba]. Soit $G$ un groupe fini. Les deux conditions suivantes sont équivalentes :

i) pour tout $G$-réseau $M, B r^{n r}\left(\mathbf{C}(M)_{0}^{G}\right)=0$;

ii) les sous-groupes de Sylow de $G$ sont abéliens bicycliques.

Nous concluons cet article par la démonstration du

Théorème. Soit $G$ un groupe fini Les deux conditions suivantes sont équivalentes:

i) pour tout $G$-réseau $M$ et toute action multiplicative tordue $f, B r^{n r}\left(\mathbf{C}(M)_{f}^{G}\right)=0$;

ii) les sous-groupes de Sylow de G sont cycliques.

Remerciements. Je tiens à remercier J.-L. Colliot-Thélène et J.-J. Sansuc qui m'ont initié au sujet, J. Lannes et Srinivas, de leurs suggestions et de leur intérêt pour ce travail. Cet article a été conçu pendant un séjour au Tata Institute of Fundamental Research de Bombay, et rédigé pendant un séjour dans la famille d'André et Catherine Bellaïche. Je remercie les deux institutions.

\section{Linéarisation des actions multiplicatives tordues}

\section{I-1. Actions multiplicatives tordues}

Soient $G$ un groupe fini, $M$ un $G$-réseau - c'est-à-dire un $\mathbf{Z}$-module libre et de type fini sur lequel $G$ agit fidèlement - et $f: G \rightarrow \widehat{M}$ un homomorphisme croisé du groupe $G$ dans le $G$-module $\widehat{M}=\operatorname{Hom}_{\mathbf{Z}}\left(M, \mathbf{C}^{*}\right)$.

On définit alors une action de $G$ dans le corps de fractions $\mathbf{C}(M)$ de l'anneau 
de groupe $\mathbf{C}[M]$ par la formule $g \circ X^{m}=f(g)(g . m) X^{g . m}$.

Cette action est dite multiplicative tordue (par $f$ ). Une autre façon utile de la définir est la suivante:

Le groupe d'automorphismes de l'anneau de groupe $\mathbf{C}[M]$ est le produit semidirect de $G L(M)$ par le $G$-module $\widehat{M}$. Nous noterons $\widetilde{G}_{M}$ l'image réciproque de $G \subset G L(M)$ dans ce produit semi-direct de sorte que l'on a la suite exacte:

$$
1 \rightarrow \widehat{M} \rightarrow \widetilde{G}_{M} \stackrel{s_{0}}{\leftrightarrows} G \rightarrow 1
$$

Faire agir $G$ dans $\mathbf{C}[M]$ par la formule $g \circ X^{m}=X^{g \cdot m}$ fournit une section canonique de la suite exacte (1) notée $s_{0}$.

La donnée d'un homomorphisme croisé $f: G \rightarrow \widehat{M}$ est équivalente à la donnée d'une seconde section $s=s_{0}+f$ de (1) et permet donc de plonger $G$ par $s$ dans $\widetilde{G}_{M}$. L'action multiplicative tordue par $f$ n'est autre que la restriction à $s(G)$ de l'action naturelle de $\widetilde{G}_{M}$.

\section{I-2. Linéarisation}

Supposons maintenant que le $G$-réseau $M$ soit d'indice fini dans un autre $G$-réseau $P$ (qui deviendra rapidement un $G$-module de permutations) et notons $F=P / M$.

Nous obtenons alors le diagramme suivant où $\widehat{F}$ est le groupe des caractères du groupe fini $F$.

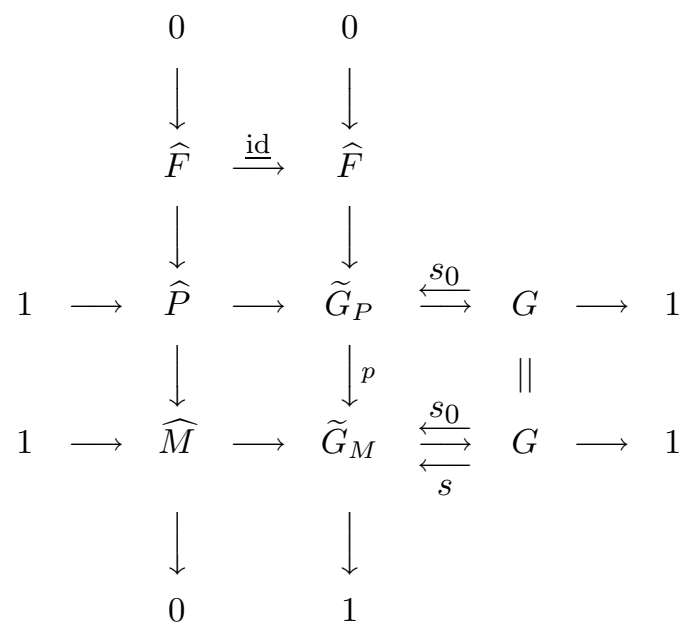

Théorème I-2.1. Notons $\mathbf{C}(M)_{f}^{G}$ le corps des invariants de $\mathbf{C}(M)$ par l'action multiplicative tordue par $f$.

i) L'extension $\mathbf{C}(P) \supset \mathbf{C}(M)_{f}^{G}$ est galoisienne ; ii) Son groupe de Galois $\Gamma$ est égal à $p^{-1}(s(G))$. 
On a donc la suite exacte:

$$
1 \longrightarrow \widehat{F} \longrightarrow \Gamma \stackrel{p}{\longrightarrow} G \longrightarrow 1
$$

iii) La classe de cette extension $x \in H^{2}(G ; \widehat{F})$ est l'image de la classe de l'homomorphisme croisé $f$ par le connectant $\delta$, de la suite exacte $0 \rightarrow \widehat{F} \rightarrow \widehat{P} \rightarrow \widehat{M} \rightarrow 0$ : $x=\delta[f]$.

iv) Si l'on suppose en outre que le $G$-réseau $P$ est un $G$-module de permutations, l'action du groupe $\Gamma$ sur le corps de fractions rationnelles $\mathbf{C}(P)$ est linéaire.

Preuve. i) et ii) Le groupe $\Gamma=p^{-1}(s(G))$, sous-groupe de $\widetilde{G}_{P}$ agit sur le corps des fractions rationelles $\mathbf{C}(P)$.

On a les égalités suivantes:

$$
\mathbf{C}(P)^{\Gamma}=\left(\mathbf{C}(P)^{\widehat{F}}\right)^{s(G)}=\mathbf{C}(M)^{s(G)}=\mathbf{C}(M)_{f}^{G} .
$$

iv) Le groupe $\widetilde{G}_{P}$ est engendré par $s_{0}(G)$ et $\widehat{P}$. Le sous-groupe $s_{0}(G)$ agit linéairement car $P$ est un $G$-module de permutations. Le sous-groupe $\widehat{P}$ agit évidemment linéairement. Il en résulte que l'action de $\widetilde{G}_{P}$, et à fortiori celle de son sous-groupe $\Gamma$, sur $\mathbf{C}(P)$ est linéaire.

iii) Soit $\tilde{f}: G \rightarrow \widehat{P}$ un relevé ensembliste de l'homomorphisme croisé $f: G \rightarrow \widehat{M}$ et soit $\tilde{s}=(\mathrm{Id}, \hat{f})$ un relevé ensembliste de $s=(\mathrm{Id}, f)$



On calcule alors $\tilde{s}\left(g_{1} g_{2}\right) \tilde{s}\left(g_{2}\right)^{-1} \tilde{s}\left(g_{1}\right)^{-1}$ et on trouve $\tilde{f}\left(g_{1} . g_{2}\right)-\tilde{f}\left(g_{1}\right)-g_{1} \cdot \tilde{f}\left(g_{2}\right)$ ce qui montre bien que l'extension $0 \rightarrow \widehat{F} \rightarrow \Gamma \rightarrow G \rightarrow 1$ a pour classe la classe $\delta[f]$.

\section{I-3. Conclusion}

A chaque action multiplicative tordue par $f$ d'un groupe fini $G$ dans un $G$-réseau $M$ d'indice fini dans un $G$-réseau de permutations, $P$, nous avons associé une action linéaire d'un groupe fini $\Gamma$ (bien déterminé par $G, M, P, f)$ dans $\mathbf{C}(P)$ de sorte que $\mathbf{C}(P)^{\Gamma}=\mathbf{C}(M)_{f}^{G}$ et donc en particulier telle que

$$
B r^{n r}\left(\mathbf{C}(P)^{\Gamma}\right)=B r^{n r}\left(\mathbf{C}(M)_{f}^{G}\right) .
$$




\section{Le théorème de Saltman [S3]}

Le but de ce paragraphe est de donner une nouvelle démonstration du théorème de Saltman ci-dessous, basée sur la linéarisation, dans le cas particulier où le $G$-réseau $M$ est d'indice fini dans un $G$-réseau de permutations $P$.

Pour exprimer ce théorème, introduisons quelques notations. Si $\Gamma$ est un groupe on note $\beta_{\Gamma}$ l'ensemble des homomorphismes du groupe $\mathbf{Z} \times \mathbf{Z}$ dans $\Gamma$. Si $U$ est un $\Gamma$-module on note $K^{i}(\Gamma ; U)$ le noyau des homomorphismes de restriction:

$$
H^{i}(\Gamma ; U) \rightarrow \prod_{\rho \in \beta_{\Gamma}} H^{i}(\operatorname{Im} \rho ; U) .
$$

Soient maintenant $G, M$ et $f: G \rightarrow \widehat{M}$ comme précédemment et $\rho \in \beta_{G}$. L'injection de $G$-modules $i: \mathbf{C}^{*} \rightarrow \mathbf{C}[M]_{f}^{*}$ induit une application

$$
i_{*}: H^{2}\left(\operatorname{Im} \rho ; \mathbf{C}^{*}\right) \rightarrow H^{2}\left(\operatorname{Im} \rho ; \mathbf{C}[M]_{f}^{*}\right) .
$$

On note $\tilde{\beta}_{G}$ le sous-ensemble de $\beta_{G}$ constitué des $\rho$ pour lesquels l'application ci-dessus est injective et pour un $G$-module $V, \widetilde{K}^{i}(G ; V)$ le noyau des homomorphismes de restriction:

$$
H^{i}(G ; V) \rightarrow \prod_{\rho \in \widetilde{\beta}_{G}} H^{i}(\operatorname{Im} \rho ; V)
$$

Théorème II-1. [S3]

$$
\operatorname{Br}^{n r}\left(\mathbf{C}(M)_{f}^{G}\right)=\widetilde{K}^{2}\left(G ; \mathbf{C}[M]_{f}^{*}\right)
$$

Grâce au théorème [Bo] rappelé dans l'introduction et à la linéarisation, le théorème précédent est équivalent au:

Théorème II-2. Soit

$$
0 \longrightarrow \widehat{F} \quad \longrightarrow \Gamma \stackrel{p}{\longrightarrow} G \longrightarrow 1 . \quad(\mathrm{e})
$$

l'extension de $G$ par le groupe des caractères du conoyau $F$ de $M$ dans $P$, d'invariant $x=\delta[f] \in H^{2}(G ; \widehat{F})$.

Alors les deux groupes

$$
\widetilde{K}^{2}\left(G ; \mathbf{C}[M]_{f}^{*}\right) \quad \text { et } \quad K^{2}\left(\Gamma ; \mathbf{C}^{*}\right)
$$

sont canoniquement isomorphes. 
La démonstration repose sur les deux lemmes fondamentaux suivants.

Lemme II-3. L'ensemble $\widetilde{\beta}_{G}$ est l'ensemble des $\rho: \mathbf{Z} \times \mathbf{Z} \rightarrow G$ tels que $\rho^{*}(x)=0$. Autrement dit, $\widetilde{\beta}_{G}$ est l'ensemble des $\rho: \mathbf{Z} \times \mathbf{Z} \rightarrow G$ qui se relèvent à $\Gamma$.

Démonstration du Lemme II-3:

Considérons la suite exacte de $G$-modules

$$
0 \longrightarrow \mathbf{C}^{*} \stackrel{i}{\longrightarrow} C[M]_{f}^{*} \longrightarrow M \longrightarrow 0
$$

La première remarque est que cette suite définit un élément dans $\operatorname{Ext}_{G}^{1}\left(M, \mathbf{C}^{*}\right)=$ $H^{1}(G ; \widehat{M})$ qui n'est autre que la classe $[f]$ de l'homomorphisme croisé $f: G \rightarrow \widehat{M}$.

Il en résulte que $\rho \in \widetilde{\beta}_{G}$ si et seulement si l'application:

$$
H^{1}(\operatorname{Im} \rho ; M) \stackrel{\cup[f]}{\longrightarrow} H^{2}\left(\operatorname{Im} \rho ; \mathbf{C}^{*}\right)
$$

est nulle.

Par ailleurs, tout élément $u \in H^{1}(\operatorname{Im} \rho ; M)$ s'écrit $\delta v$ où $v \in H^{0}(\operatorname{Im} \rho ; F)=$ $H^{0}(\mathbf{Z} \times \mathbf{Z} ; F)$.

(Puisque $H^{1}(\operatorname{Im} \rho ; P)$ est nul, car $P$ est un $G$-module de permutations).

L'application $\rho^{*}: H^{2}\left(\operatorname{Im} \rho ; \mathbf{C}^{*}\right) \rightarrow H^{2}\left(\mathbf{Z} \times \mathbf{Z} ; \mathbf{C}^{*}\right)$ est injective puisque elle est duale de l'application $\rho_{*}: H_{2}(\mathbf{Z} \times \mathbf{Z} ; \mathbf{Z}) \cong \Lambda^{2} \mathbf{Z} \rightarrow H_{2}(\operatorname{Im} \rho ; \mathbf{Z}) \cong \Lambda^{2}(\operatorname{Im} \rho)$ évidemment surjective.

On arrive donc à l'équivalence :

$$
\rho \in \widetilde{\beta}_{G} \Longleftrightarrow \delta v \cup \rho^{*}([f])=0 \quad \forall v \in H^{0}(\mathbf{Z} \times \mathbf{Z} ; F)
$$

ou encore

$$
\begin{aligned}
& \Longleftrightarrow v \cup \rho^{*}(\delta[f])=0 \quad \forall v \in H^{0}(\mathbf{Z} \times \mathbf{Z} ; F) \\
& \Longleftrightarrow v \cup \rho^{*}(x)=0 \quad \forall v \in H^{0}(\mathbf{Z} \times \mathbf{Z} ; F)
\end{aligned}
$$

Il suffit maintenant de remarquer que le cup-produit:

$$
H^{0}(\mathbf{Z} \times \mathbf{Z} ; F) \times H^{2}(\mathbf{Z} \times \mathbf{Z} ; \widehat{F}) \rightarrow H^{2}\left(\mathbf{Z} \times \mathbf{Z} ; \mathbf{C}^{*}\right)
$$

est non dégénéré. En effet, la dualité de Poincaré du groupe $\mathbf{Z} \times \mathbf{Z}$ identifie cet accouplement à:

$$
\begin{array}{ccccc}
H^{0}(\mathbf{Z} \times \mathbf{Z} ; F) & \times & H_{0}(\mathbf{Z} \times \mathbf{Z} ; \widehat{F}) & \rightarrow & H^{2}\left(\mathbf{Z} \times \mathbf{Z} ; \mathbf{C}^{*}\right) \\
\| & & \| & & \| \\
F^{G} & \times & \widehat{F}_{G} & \rightarrow & \mathbf{C}^{*}
\end{array}
$$


Lemme II-4. Soit $\rho: \mathbf{Z} \times \mathbf{Z} \rightarrow G, \quad \rho \in \widetilde{\beta}_{G}$, et considérons le pull-back de l'extension (e) par $\rho$.

On obtient le diagramme

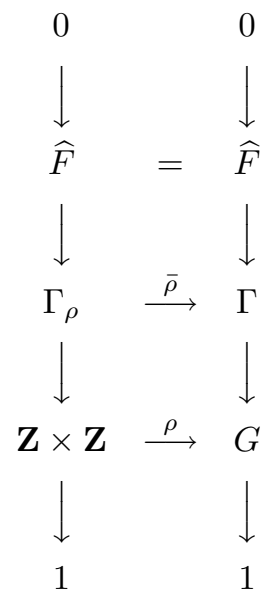

où d'après le Lemme II.3, $\Gamma_{\rho}$ est le produit semi-direct de $\mathbf{Z} \times \mathbf{Z}$ et de $\widehat{F}$. Alors

$$
K^{2}\left(\Gamma_{\rho} ; \mathbf{C}^{*}\right)=0 .
$$

Ce lemme est le phénomène essentiel. Sa démonstration est donnée dans [BaTh3].

Considérons maintenant la suite exacte des termes de bas degré pour l'extension (e) et pour toutes les extensions scindées $\rho^{*}(e), \rho \in \widetilde{\beta}_{G}$. Nous obtenons le diagramme ci-après:

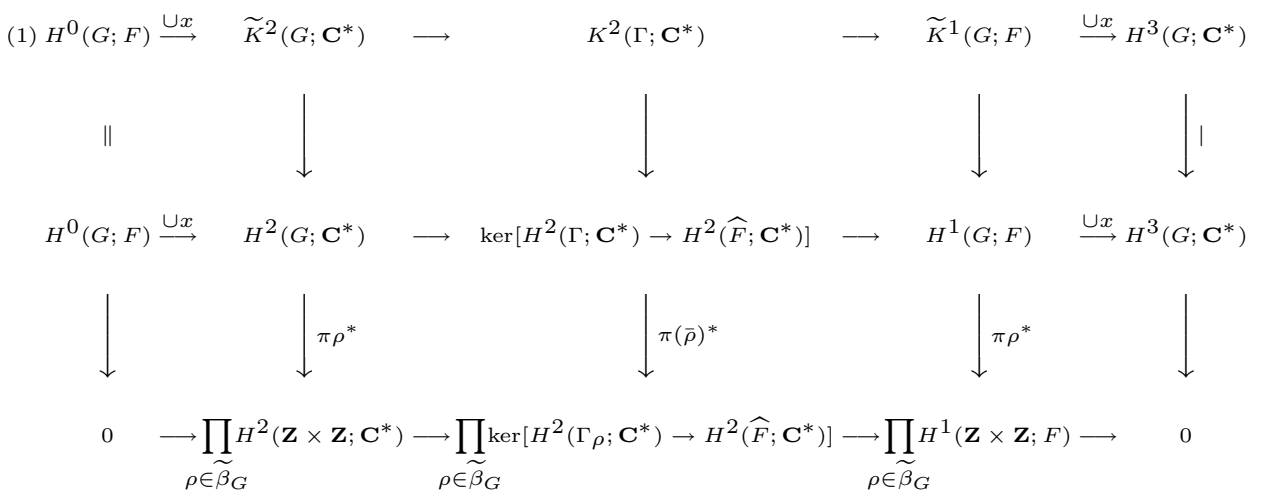

Soit $\rho: \mathbf{Z} \times \mathbf{Z} \rightarrow \Gamma$ un homomorphisme. Comme mentionné précédemment l'application 
$H^{2}\left(\operatorname{Im} \rho ; \mathbf{C}^{*}\right) \stackrel{\rho^{*}}{\rightarrow} H^{2}\left(\mathbf{Z} \times \mathbf{Z} ; \mathbf{C}^{*}\right)$ étant injective, le groupe $K^{2}\left(\Gamma ; \mathbf{C}^{*}\right)$ est donc aussi constitué des classes de cohomologie $x \in H^{2}\left(\Gamma ; \mathbf{C}^{*}\right)$ telles que $\rho^{*}(x) \in H^{2}(\mathbf{Z} \times \mathbf{Z} ; \Gamma)$ soit nulle pour tout $\rho \in \beta_{\Gamma}$.

Le lemme II-4 montre alors que le noyau de l'homomorphisme $\prod \bar{\rho}^{*}$ évidemment inclus dans $K^{2}\left(\Gamma ; \mathbf{C}^{*}\right)$ lui est en fait égal et on obtient la suite (1) qui est exacte sauf peut-être en $\widetilde{K}^{1}(G ; F)$.

Par ailleurs, la suite $0 \rightarrow \mathbf{C}^{*} \rightarrow \mathbf{C}[M]_{f}^{*} \rightarrow M \rightarrow 0$ conduit à la suite

$$
\begin{aligned}
H^{1}(G ; M) & \stackrel{\cup[f]}{\rightarrow} \widetilde{K}^{2}\left(G ; \mathbf{C}^{*}\right) \rightarrow \widetilde{K}^{2}\left(G ; \mathbf{C}[M]_{f}^{*}\right) \\
& \rightarrow \widetilde{K}^{2}(G ; M) \stackrel{\cup[f]}{\rightarrow} H^{3}\left(G ; \mathbf{C}^{*}\right)
\end{aligned}
$$

qui est exacte sauf peut-être en $\widetilde{K}^{2}(G ; M)$.

Soit $\delta$ le bord de la suite exacte de $G$-modules

$$
0 \rightarrow M \rightarrow P \rightarrow F \rightarrow 0
$$

Nous obtenons le diagramme commutatif

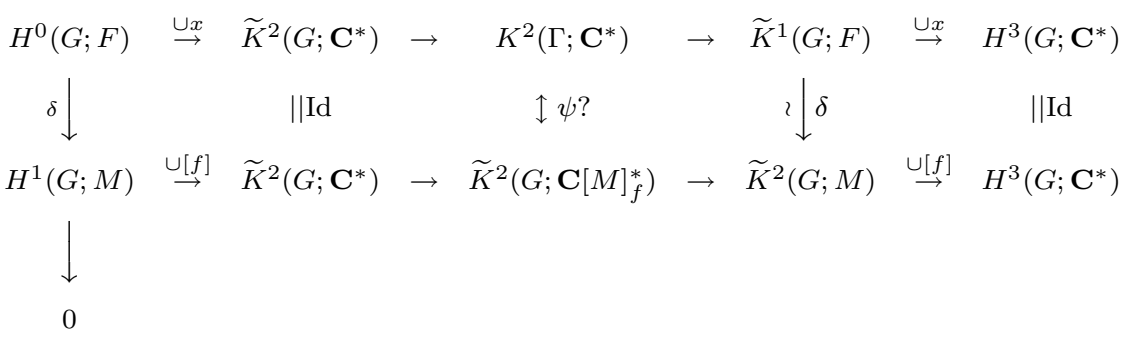

où le premier $\delta$ vertical à gauche est surjectif (puisque $H^{1}(G, P)=0$ ) tandis que le second $\delta$ vertical est un isomorphisme.

Pour montrer ce fait il suffit de voir que $\widetilde{K}^{2}(G ; P)=0$. Nous avons la suite d'inclusions

$$
\widetilde{K}^{2}(G ; P) \subset H^{2}(G, P)=\underset{H}{\oplus} H^{2}(G ; \mathbf{Z}[G / H])=\underset{H}{\oplus} H^{2}(H, \mathbf{Z})=\underset{H}{\oplus} H^{1}(H ; Q / Z)
$$

Par ailleurs tout sous-groupe cyclique de $G$ est l'image d'un $\rho: \mathbf{Z} \times \mathbf{Z} \rightarrow G$, $\rho \in \tilde{\beta}_{G}$.

Ceci montre que $\widetilde{K}^{2}(G ; P)$ est contenu dans le noyau

$$
\begin{aligned}
& \oplus \operatorname{ker}\left(H^{1}(H ; Q / \mathbf{Z}) \rightarrow H^{1}(C, Q / \mathbf{Z})\right) \\
& H
\end{aligned}
$$

pour tout sous-groupe cyclique $C$ de $H$. Ce dernier groupe est bien nul. 
Soient $I$ l'image de $K^{2}\left(\Gamma ; \mathbf{C}^{*}\right)$ dans $\widetilde{K}^{1}(G ; F)$ et $I^{\prime}$ celle de $\widetilde{K}^{2}\left(G ; \mathbf{C}[M]_{f}^{*}\right)$ dans $\widetilde{K}^{2}(G ; M)$.

On a les suites exactes

$$
\begin{array}{cccccc}
0 & \rightarrow \quad I & \rightarrow & \operatorname{ker}(\cup x) & \rightarrow & \prod_{\rho \in \tilde{\beta}_{G}} H^{2}\left(\mathbf{Z} \times \mathbf{Z} ; \mathbf{C}^{*}\right) / H^{2}\left(G ; \mathbf{C}^{*}\right) \\
& \delta \downarrow & & \delta \downarrow & & \| \mathrm{Id} \\
0 & \rightarrow \quad I^{\prime} & \rightarrow & \operatorname{ker}(\cup[f]) & \rightarrow & \prod_{\rho \in \tilde{\beta}_{G}} H^{2}\left(\mathbf{Z} \times \mathbf{Z} ; \mathbf{C}^{*}\right) / H^{2}\left(G ; \mathbf{C}^{*}\right)
\end{array}
$$

ce qui montre que l'isomorphisme $\delta: \widetilde{K}^{1}(G ; F) \stackrel{\sim}{\rightarrow} \widetilde{K}^{2}(G ; \Gamma)$ identifie $I$ et $I^{\prime}$.

On a donc le diagramme suivant à lignes exactes

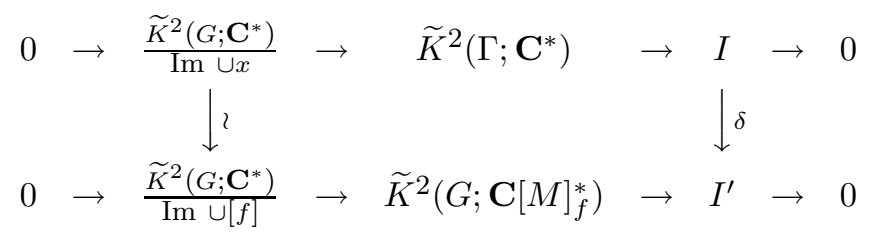

Pour conclure il manque un homomorphisme (et donc un isomorphisme) compatible $\psi: K^{2}\left(\Gamma ; \mathbf{C}^{*}\right) \rightarrow \widetilde{K}^{2}\left(G ; \mathbf{C}[M]_{f}^{*}\right)$.

En fait nous avons le

\section{Lemme II-5.}

i) L'application $i_{*}: K^{2}\left(\Gamma ; \mathbf{C}^{*}\right) \rightarrow H^{2}\left(\Gamma ; \mathbf{C}[M]_{f}^{*}\right)$ est injective.

ii) L'application $p^{*}: \widetilde{K}^{2}\left(G ; \mathbf{C}[M]_{f}^{*}\right) \rightarrow H^{2}\left(\Gamma ; \mathbf{C}[M]_{f}^{*}\right)$ est injective.

iii) $\operatorname{Im} i_{*}=\operatorname{Im} p^{*}$.

Preuve.

i) En fait nous allons montrer que $H^{2}\left(\Gamma ; \mathbf{C}^{*}\right) \stackrel{i_{*}}{\rightarrow} H^{2}\left(\Gamma ; \mathbf{C}[M]_{f}^{*}\right)$ est injective.

On a le diagramme suivant

$$
H^{0}(\Gamma ; F) \stackrel{d}{\rightarrow} H^{1}(\Gamma ; M) \stackrel{\delta}{\rightarrow} H^{2}\left(\Gamma ; \mathbf{C}^{*}\right)
$$

où $d$ est le bord de la suite exacte de $\Gamma$-modules $0 \rightarrow M \rightarrow P \rightarrow F$ et $\delta$ celui de la suite exacte de $\Gamma$-module $0 \rightarrow \mathbf{C}^{*} \rightarrow \mathbf{C}[M]_{f}^{*} \rightarrow M \rightarrow 0$.

La composée $\delta \circ d$ est le cup produit par la classe $p^{*}(x) \in H^{2}(\Gamma ; \widehat{F})$. Cette classe est tautologiquement nulle. Par ailleurs l'application $d$ est surjective (puisque $\left.H^{1}(\Gamma, P)=0\right)$. On en conclut que $\delta$ est nulle ou encore que $i_{*}$ est injective.

ii) Là encore nous allons montrer que

$$
p^{*}: H^{2}\left(G ; \mathbf{C}[M]_{f}^{*}\right) \rightarrow H^{2}\left(\Gamma ; \mathbf{C}[M]_{f}^{*}\right)
$$

est injective ou de façon équivalente que

$$
\cup x: H^{0}\left(G ; H^{1}\left(\widehat{F} ; \mathbf{C}[M]_{f}^{*}\right)\right) \rightarrow H^{2}\left(G ; \mathbf{C}[M]_{f}^{*}\right)
$$


est nulle.

On a le diagramme commutatif suivant:

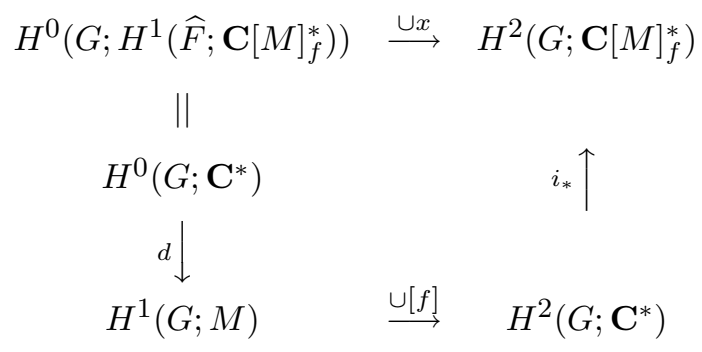

et $i_{*} \circ \cup[f]=0$.

iii) Nous laissons le lecteur se convaincre de ce point.

\section{Un exemple}

Le but de ce paragraphe est de donner, pour tout nombre premier $p$, un exemple d'action multiplicative tordue du groupe $G=\mathbf{Z} / p \times \mathbf{Z} / p$ dans un $G$-réseau $M$, telle que le groupe de Brauer non ramifié, $B r^{n r} \mathbf{C}(M)_{f}^{G}$ soit non nul.

Comme suggéré précédemment, il revient au même de construire une extension :

$$
0 \rightarrow \widehat{F} \rightarrow \Gamma \rightarrow G \rightarrow 1
$$

telle que $K^{2}\left(\Gamma ; \mathbf{C}^{*}\right)$ soit non nul. En effet, on choisit alors pour $G$-réseau $M$ le noyau de n'importe quelle surjection d'un $G$-module libre $L$ sur $F$, et un homomorphisme croisé $f: G \rightarrow \widehat{M}$, tel que $\delta[f]=[e] \in H^{2}(G ; \widehat{F})$ où $\delta$ est le connectant de la suite exacte de $G$-module

$$
0 \rightarrow \widehat{F} \rightarrow \widehat{L} \rightarrow \widehat{M} \rightarrow 0
$$

(observer que $H^{2}(G ; \widehat{L})=0$ ). Ainsi on aura bien l'égalité

$$
K^{2}\left(\Gamma ; \mathbf{C}^{*}\right)=\widetilde{K}^{2}\left(G ; \mathbf{C}[M]_{f}^{*}\right)=B r^{n r}\left(\mathbf{C}(M)_{f}^{G}\right) .
$$

Nous allons réaliser les 2 conditions suivantes:

1) choisir (e) "assez compliquée" pour que tout $\rho: \mathbf{Z} \times \mathbf{Z} \rightarrow G=\mathbf{Z} / p \times \mathbf{Z} / p$, $\rho \in \widetilde{\beta}_{G}$, soit non surjectif

2) choisir $F$ tel que $H^{1}(G ; F)$ soit isomorphe à $\mathbf{Z} / p^{2}$.

Soit alors $x$ un générateur du groupe cyclique $H^{1}(G ; F)$ et considérons l'élément $y=p x \neq 0 \in H^{1}(G ; F)$. 
Puisque $H^{3}\left(G ; \mathbf{C}^{*}\right)$ est tué par $p$, il existe $z \in H^{2}\left(\Gamma ; \mathbf{C}^{*}\right)$ qui relève l'élément $y$ et qui est donc en particulier non nul.

La condition (1) montre que pour tout $\rho: \mathbf{Z} \times \mathbf{Z} \rightarrow \Gamma$ nous avons le diagramme commutatif ci-dessous

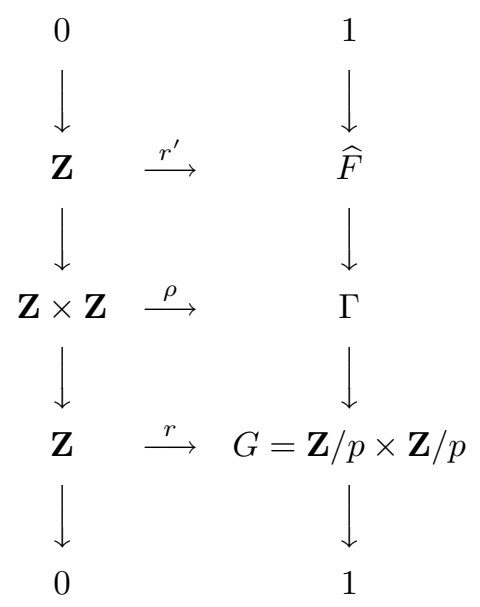

d'où le diagramme commutatif

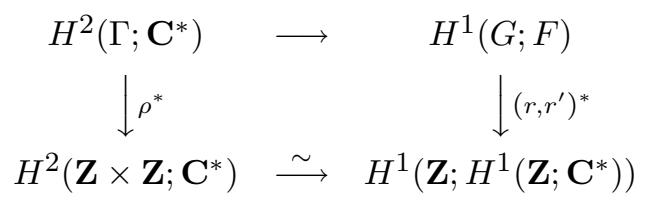

qui montre que $\rho^{*}(z)=\left(r, r^{\prime}\right)^{*}(y)$.

Ce dernier élément est nul car l'homomorphisme $r$ transite par un sous-groupe d'ordre au plus $p$. Ce qui montre que l'élément $z \neq 0$ appartient bien à $K^{2}\left(\Gamma ; \mathbf{C}^{*}\right)$.

Il reste à réaliser les conditions 1) et 2).

Posons $F=\frac{I[G]}{p^{2}}$ où $I[G]$ est l'idéal d'augmentation de l'anneau de groupe $\mathbf{Z}[G]$.

\section{Lemme III-1.}

$$
\begin{aligned}
& H^{1}(G ; F) \simeq \mathbf{Z} / p^{2} \\
& H^{1}(G ; \widehat{F}) \simeq(\mathbf{Z} / p)^{2} \\
& H_{0}(G ; \widehat{F}) \simeq \mathbf{Z} / p^{2}
\end{aligned}
$$

Preuve. Exercice.

La condition 1) résulte du : 
Lemme III-2. Soit $s: \mathbf{Z} \times \mathbf{Z} \rightarrow \mathbf{Z} / p \times \mathbf{Z} / p$ la surjection canonique. Il existe une extension

$$
0 \rightarrow \frac{\widehat{I[G]}}{p^{2}} \rightarrow \Gamma \rightarrow \mathbf{Z} / p \times \mathbf{Z} / p=G \rightarrow 1
$$

telle que l'extension $s^{*}(e)$ soit non triviale.

Avant de donner la preuve de ce lemme, montrons comment il entraîne la condition 1).

Soit donc $\rho: \mathbf{Z} \times \mathbf{Z} \rightarrow \mathbf{Z} / p \times \mathbf{Z} / p$ un homomorphisme surjectif et $\bar{\rho}$ un relevé à $\mathbf{Z} \times \mathbf{Z}, \rho=s \circ \bar{\rho}$.

$$
\begin{array}{ccc}
\mathbf{Z} \times \mathbf{Z} & \stackrel{s}{\longrightarrow} & \mathbf{Z} / p \times \mathbf{Z} / p \\
& \backslash \bar{\rho} & \uparrow \rho \\
& & \mathbf{Z} \times \mathbf{Z}
\end{array}
$$

Puisque $\rho$ est surjectif, $\bar{\rho}$ est injectif et l'indice de $\bar{\rho}(\mathbf{Z} \times \mathbf{Z})$ dans $\mathbf{Z} \times \mathbf{Z}$ est premier à $p$.

Il en résulte que $\bar{\rho}^{*}: H^{2}(\mathbf{Z} \times \mathbf{Z} ; \widehat{F}) \rightarrow H^{2}(\mathbf{Z} \times \mathbf{Z} ; \widehat{F})$ est un isomorphisme, donc que $\rho^{*}(e)$ est non triviale et $\rho \notin \widetilde{\beta}_{G}$.

Preuve du Lemme III-2. La suite exacte courte

$$
0 \longrightarrow K=\mathbf{Z} \times \mathbf{Z} \stackrel{p}{\longrightarrow} \mathbf{Z} \times \mathbf{Z} \stackrel{s}{\longrightarrow} G=\mathbf{Z} / p \times \mathbf{Z} / p \quad \longrightarrow 0
$$

induit en cohomologie la suite exacte

$$
H^{2}(G ; \widehat{F}) \stackrel{s^{*}}{\longrightarrow} \operatorname{ker}\left[H^{2}(\mathbf{Z} \times \mathbf{Z} ; \widehat{F}) \stackrel{(p \times \mathrm{Id})^{*}}{\longrightarrow} H^{2}(K ; \widehat{F})\right] \stackrel{\delta}{\longrightarrow} H^{1}\left(G ; H^{1}(K ; \widehat{F})\right)
$$

Il faut montrer que l'application $s^{*}$ est non nulle ou donc que l'application $\delta$ est non injective. En fait, le domaine de définition de l'homomorphisme $\delta$ est isomorphe aux invariants sous $G$ du $G$-module $\widehat{F}$, groupe isomorphe à $\mathbf{Z} / p^{2}$, tandis qu'il prend ses valeurs dans un groupe tué par $p$. (Lemme III-1)

\section{IV.}

Le but de ce paragraphe est la démonstration du théorème suivant:

Théorème IV-1. Soit $G$ un groupe fini. Les deux conditions suivantes sont équivalentes: 
i) Les sous-groupes de Sylow du groupe $G$ sont cycliques.

ii) Pour tout $G$-réseau $M$ et toute action multiplicative tordue (par $f$ ), le groupe de Brauer non ramifié $\operatorname{Br}^{n r}\left(\mathbf{C}(M)_{f}^{G}\right)$ est nul.

Preuve. $\mathrm{i} \Rightarrow$ ii.

Lorsque $M$ est d'indice fini dans un $G$-module de permutations, cela résulte du théorème II-1, en observant que la cohomologie d'un groupe s'injecte dans le produit de celle de ses sous-groupes de Sylow, et du fait déjà utilisé précédemment que tout sous-groupe cyclique de $G$ est l'image d'un certain $\rho$ appartenant à $\widetilde{\beta}_{G}$.

Le cas général où le $G$-réseau $M$ n'est plus nécessairement d'indice fini dans un $G$-réseau de permutations résulte des deux faits suivants.

Fait 1: Soit $M$ un $G$-réseau. Alors il existe un $G$-réseau $N$ tel que $M \oplus N$ soit d'indice fini dans un $G$-réseau de permutations.

Fait 2: Si $M$ et $N$ sont deux $G$-réseaux et $f: G \rightarrow \widehat{M}$ un homomorphisme croisé, le groupe $\operatorname{Br}^{n r}\left(\mathbf{C}(M)_{f}^{G}\right)$ s'injecte dans $\operatorname{Br}^{n r}\left(\mathbf{C}(M \oplus N)_{f \oplus 0}^{G}\right)$ où 0 désigne l'homomorphisme croisé nul de $G$ dans $\widehat{N}$.

Nous laissons la démonstration du fait 1 en exercice.

Preuve du fait 2: On observe d'abord que puisque $\mathbf{C}(M)$ est transcendant pur sur $\mathbf{C}$, le groupe $B r^{n r}\left(\mathbf{C}(M)_{f}^{G}\right)$ est en fait un sous-groupe du groupe de Brauer relatif, à savoir $H^{2}\left(G ; \mathbf{C}(M)_{f}^{*}\right)$. Il reste à voir que ce groupe de cohomologie s'injecte dans $H^{2}\left(G ; \mathbf{C}(M \oplus N)_{f \oplus 0}^{*}\right)$.

Pour cela considérons la suite d'injections de $G$-modules :

$$
\mathbf{C}(M)_{f}^{*} \stackrel{i_{1}}{\hookrightarrow}\left(\mathbf{C}(M)_{f}[N]\right)^{*} \stackrel{i_{2}}{\hookrightarrow}\left(\mathbf{C}(M \oplus N)_{f \oplus 0}\right)^{*}
$$

La première admet une $G$-retraction (induite par l'homomorphisme de $N$ dans le groupe trivial) et donc induit une injection en cohomologie. Quant à la deuxième

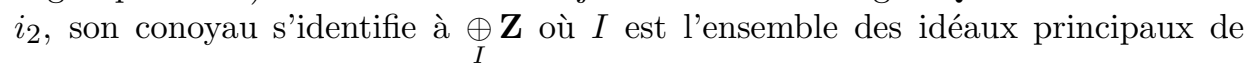
l'anneau factoriel $\mathbf{C}(M)_{f}[N]$. Ces idéaux sont permutés par $G$ et donc $H^{1}(G ; \underset{I}{\oplus} \mathbf{Z})=$ 0 , ce qui montre que $H^{2}\left(G ;\left(\mathbf{C}(M)_{f}[N]\right)^{*}\right)$ s'injecte dans $H^{2}\left(G ;\left(\mathbf{C}(M \oplus N)_{f \oplus 0}\right)^{*}\right)$. ii $\Rightarrow$ i.

- Nous savons déjà [Ba-Th.4] que les sous-groupes de Sylow de $G$ doivent être abéliens bicycliques pour que le groupe de Brauer non ramifié du corps d'invariants de toute action multiplicative non tordue soit nul.

- Si $G$ possède un $p$ sous-groupe de Sylow abélien bicyclique, non cyclique, il contient un sous-groupe $H$ isomorphe à $\mathbf{Z} / p \times \mathbf{Z} / p$. Il est alors facile "d'induire de $H$ à $G$ " l'exemple de la partie III pour produire un $G$-réseau $M$ et un homomorphisme croisé $f: G \rightarrow \widehat{M}$ tels que $\operatorname{Br}^{n r}\left(\mathbf{C}(M)_{f}^{G}\right)$ soit non nul. Nous ne détaillerons pas cette construction. 


\section{Bibliographie}

[Ba] J. Barge, Cohomologie des groupes et corps d'invariants multiplicatifs. Math. Ann. 283 (1989), 519-528.

[Bo] F. A. Bogomolov, The Brauer group of quotient spaces by linear group actions. Izv. Akad. Nauk. Sci. Mat. 51 (1987), 485-516.

[CT] J.-L. Colliot-Thélène (with the collaboration of J.-J. Sansuc), The rationality problem for fields of invariants under linear algebraic groups (with special regards to the Brauer group) IX Escuela Latinoamericana de mathemàticas, Santiago (Chili) July (1988).

[S1] D.-J. Saltman, Noether's problem over an algebraically closed field. Invent. Math. $\mathbf{7 7}$ (1984), 71-84.

[S2] D.-J. Saltman, Multiplicative field invariants. J. Algebra 106 (1987), 221-238.

[S3] D. J. Saltman, Multiplicative field invariants and the Brauer group. J. Algebra 133 (1990), $533-544$.

Jean Barge

F-91128 Palaiseau Cedex

Phone: 33169334088

Fax: 33169333019

e-mail: secret@orphee.polytechnique.fr

(Received: October 13, 1992; July 3, 1996) 